\title{
Research of Image Segmentation Algorithm Based on Edge Detection
}

\author{
Yue Liu, Mei-shan Li *, Chun-yan Fu \\ College of Information Science \&Electronic Technology, Jiamusi University, Jiamusi, 154007, China \\ e-mail:liuyue197612@126.com, friend9023@sina.com,jmsfu@163.com
}

${ }^{*}$ Corresponding author

Keywords: Image Segmentation; Edge Detection; Gradient Operator; Laplacian Operator

\begin{abstract}
This Image segmentation is the key step of image processing transitional to image analysis. Edge detection is the most commonly used method to segment images based on gray mutation. This paper mainly introduces the method of edge detection to gradient operator and Laplacian operator.
\end{abstract}

\section{Introduction}

Image segmentation is the key step of image processing transitional to image analysis. So the effect of image segmentation will directly influence the understanding of images. Edge detection is an important way of image segmentation. Different images have different gray scales, and there usually are obvious edges at the border. We can segment images based on these features. The commonly used technology is to calculate the gradient operator and Laplace operator ${ }^{[1]}$. This paper will mainly introduce three kinds of gradient operators and the second difference operators.

\section{Gradient and gradient operator}

Gradient belongs to first order differential operator, and corresponds to the first derivative. If images contain less noise and the grey value transition at the image border is more obvious, gradient operator can obtain a better effect of edge detection. Several common used and typical differential operator methods, such as Roberts operator, Sobel operator and Prewitt operator, all take advantage of the method to detect the maximum of gradient ${ }^{[2]}$.

\section{Roberts Operator}

Roberts edge detection operator is a kind of operator which detects edges by means of local difference operator.

Its expression is:

$$
R(x, y)=\sqrt{[f(x, y)-f(x+1, y+1)]^{2}+[f(x+1, y)-f(x, y+1)]^{2}}
$$

Also it may be approximately expressed: $R(x, y)=|f(x, y)-f(x+1, y+1)|+\mid f(x+1, y)-f(x, y+1)$

Besides, it may be represented by means of the maximum of the Roberts operator:

$$
R(x, y)=\max (|f(x, y)-f(x+1, y+1)|,|f(x, y+1)-f(x+1, y)|)
$$

The edges obtained from the above expressions are of a better edge orientation invariance. In the case that the length of edges is equal but with different orientation, the change of synthetic amplitude obtained via the maximum of the Roberts operator is smaller than that via Roberts crossover operator. Its convolution template is as shown in Figure 1.

$$
\left(\begin{array}{rr}
1 & 0 \\
0 & -1
\end{array}\right) \quad\left(\begin{array}{rr}
0 & 1 \\
-1 & 0
\end{array}\right)
$$

Fig. 1 Roberts operator template

It is an operation on a $2 \times 2$ template ${ }^{[3]}$. After evaluating the gradient value of all pixels in the images expressing by Roberts operator respectively, an appropriate threshold value needs to be 
defined. When marking the gradient values greater than the threshold as 1 and less than the threshold as 0 , the edge images can be obtained by means of Roberts algorithm. The Figure 2 is the original image, and the Figure 3 is the image segmented by Roberts operator.

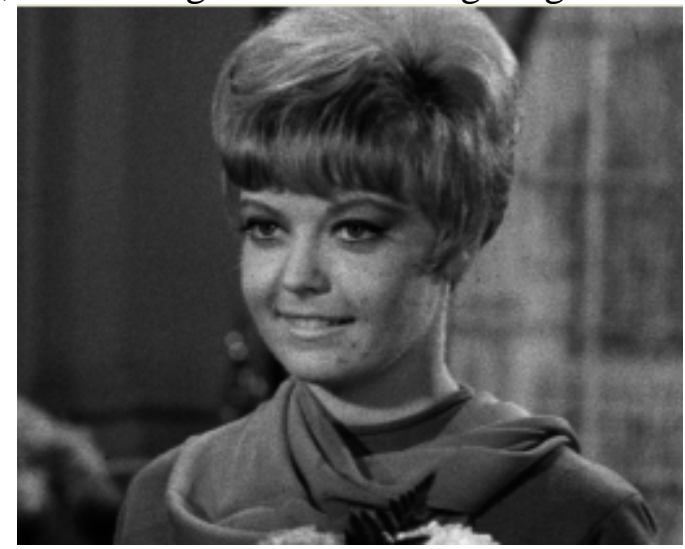

Fig. 2 original image

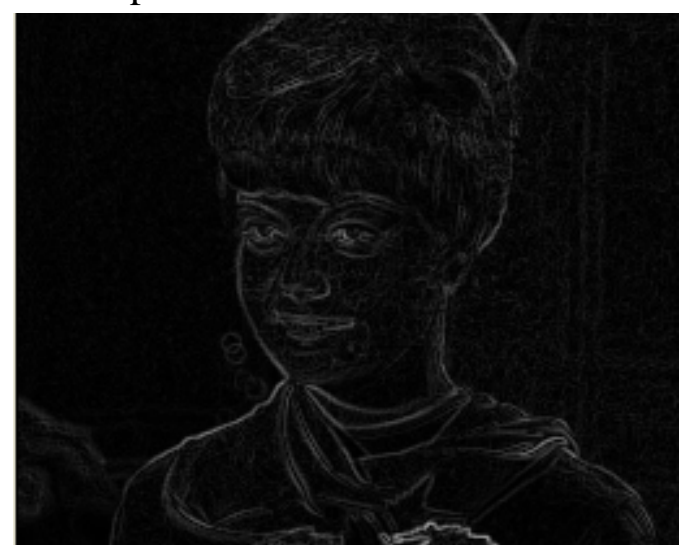

Fig. 3 the image processed by Roberts edge detection.

Roberts operator performs an accurate edge positioning, but is sensitive to the noise ${ }^{[4]}$. So it is suitable for image segmentation when the images have obvious edges and less noise. Such as the binary image.

\section{Sobel Operator}

The Sobel operator is a partial derivative which calculates the $\mathrm{x}$ direction and y direction of the 3 $\mathrm{x} 3$ neighborhood with the center $\mathrm{f}(\mathrm{x}, \mathrm{y})$, and set up the weightingon the central position of pixels ${ }^{[5]}$, namely:

$$
\begin{aligned}
& S x=\{f(x-1, y+1)+2 f(x, y+1)\}+f(x+1, y+1)-\{f(x-1, y-1)+2 f(x, y-1)+(x+1, y-1)\} \\
& S y=\{f(x+1, y-1)+2 f(x+1, y)\}+f(x+1, y+1)-\{f(x-1, y-1)+2 f(x-1, y)+f(x-1, y+1)\}
\end{aligned}
$$

Above expression adopts the method of combining the weighted mean difference of $f(x, y)$ neighborhood image intensity, split operation and local average. Its gradient value is:

$$
g(x, y)=\sqrt{S x^{2}+S y^{2}}
$$

The convolution template of Sobel algorithm is:

$$
S x=\left(\begin{array}{lll}
1 & 0 & 1 \\
2 & 0 & 2 \\
1 & 0 & 1
\end{array}\right) \quad S y=\left(\begin{array}{lll}
1 & 2 & 1 \\
0 & 0 & 0 \\
1 & 2 & 1
\end{array}\right)
$$

Fig. 4 Sobel operator template

Using the above two operators to conduct image operations and substituting into the expression (6), the gradient amplitude value $\mathrm{g}(\mathrm{x}, \mathrm{y})$ of the images can be obtained. Then define the appropriate threshold $\mathrm{T}$, so that the pixel whose gradient value $\mathrm{R}(\mathrm{x}, \mathrm{y})>\mathrm{T}$ is set as 1 , otherwise as $0 .\{\mathrm{g}(\mathrm{x}, \mathrm{y})\}$ is a binary image, namely the edge images are as shown in Figure 5.

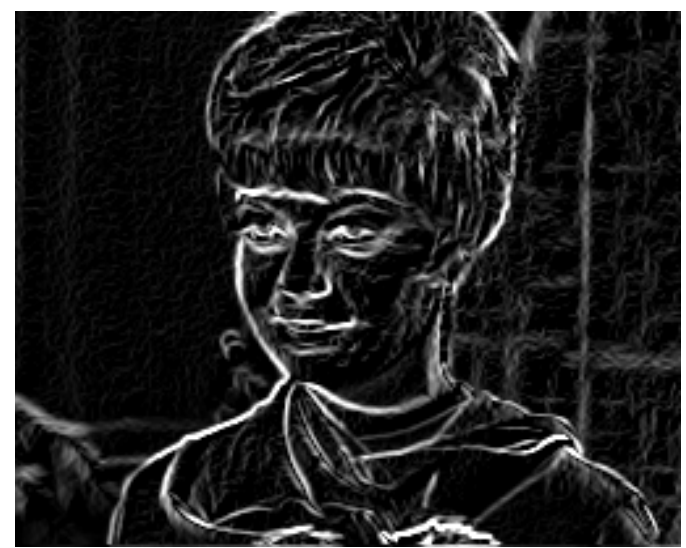

Fig. 5 the image processed by Sobel egde detection 


\section{Prewitt Operator}

Prewitt operator is an edge template operator ${ }^{[6]}$. Every kind of operator template corresponds with a kind of ideal edge sub-image. Using these operators to conduct edge detection to images successively, the obtained maximum operator template is most similar with the tested area. Also the gray value of the edge pixel may be calculated by the operator. Prewitt calculated the difference operator by expanding the size of the operator template from $2 \times 2$ to $3 \times 3$, and proposed Prewitt operator. This operator combines the local average and the direction differential-phase operation. The expression of Prewitt operator is:

$$
\begin{aligned}
& f_{x}=\{f(x-1, y+1)+f(x, y+1)\}+f(x+1, y+1)-\{f(x-1, y-1)+f(x, y-1)+(x+1, y-1)\} \\
& f_{y}=\{f(x+1, y-1)+f(x+1, y)\}+f(x+1, y+1)-\{f(x-1, y-1)+f(x-1, y)+f(x-1, y+1)\}
\end{aligned}
$$

The convolution template of Prewitt operator is:

$$
\left(\begin{array}{lll}
-1 & 0 & 1 \\
-1 & 0 & 1 \\
-1 & 0 & 1
\end{array}\right) \quad\left(\begin{array}{rrr}
1 & 1 & 1 \\
0 & 0 & 0 \\
-1 & -1 & -1
\end{array}\right)
$$

Fig. 6 Prewitt operator template

Define an appropriate threshold Tk, and make the following judgment: $P(\mathrm{i}, \mathrm{j})>\mathrm{Tk}$ is a stepjumping-like edge points. $\{\mathrm{P}(\mathrm{i}, \mathrm{j})\}$ is the edge image. As shown in Figure 7 is the image processed by Prewitt edge detection.

The difference of Prewitt operator from Sobel operator lies in that it doesn't lay stress on the pixels close to the template center. When an edge detector consists of two convolution operators, the larger value is usually taken as the end value. But it causes the sensitivity of convolution operator to the direction of edge point ${ }^{[7]}$. If it requires all-around response with more consistent performance, the radication of its quadratic sum should be taken, which is more close to the real gradient value. Alternatively, it is feasible to expand a small amount of directions operator templates of Prewitt operators to eight-direction edge operator templates, and these operator templates are also made of ideal edge sub-images. Obtain the output value of operators $p(i, j)$ by using edge templates in turn to detect images, and provide the template of the maximum value which is most close to the tested area, thus the edge pixels can be detected. Eight-direction Prewitt edge detection operator templates and their respective edge direction definitions are shown as Figures 8, and 9.
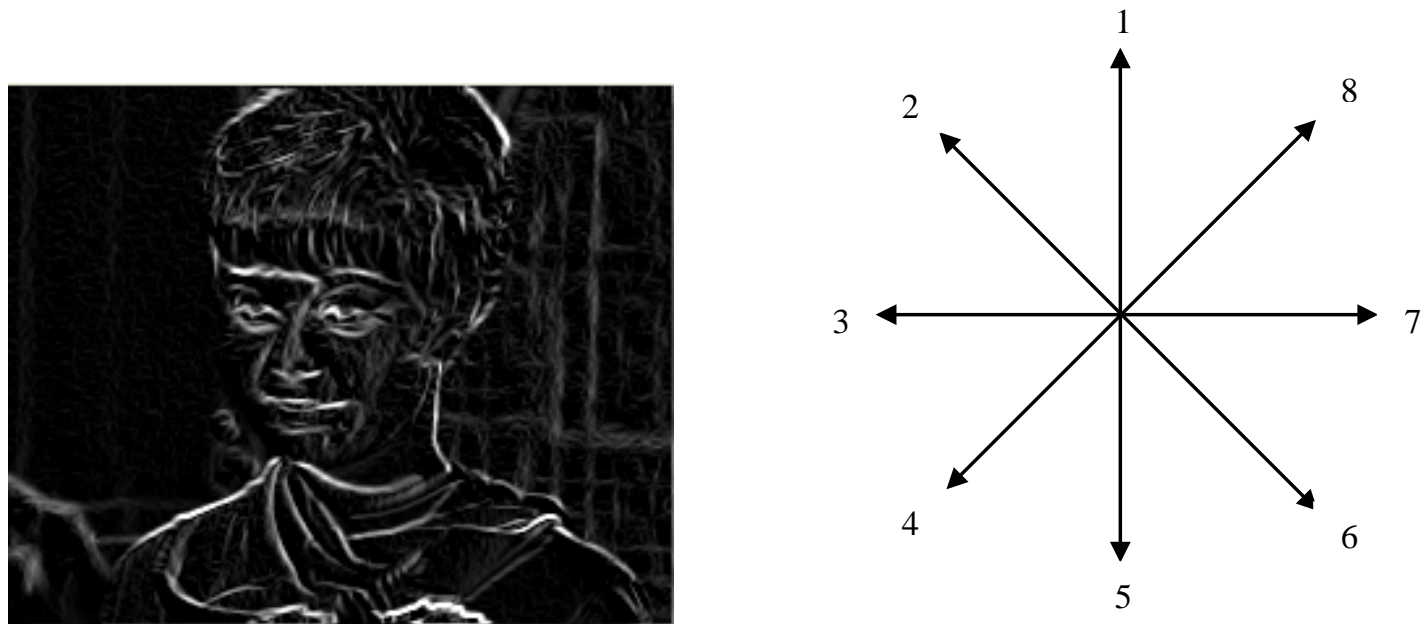

Fig. 7 the image processed by Prewitt edge detection Fig. 8 the edge directions of eight-direction operator templates

\section{Second-difference Operator}

Laplacian operator is Second-difference Operator ${ }^{[8]}$.Laplacian operator belongs to second order differential operator. It is isotropic, namely, has nothing to do with the direction of axis, coordinate axis of rotation, the results of gradient unchanged. However, the more sensitive to 
noise, therefore, Usually the first smoothed image, smoothing is used as template, so the usual segmentation algorithms are the Laplacian operator and smoothing operator together to create a new template. Expression of the Laplacian operator for:

$$
\nabla^{2} f(x, y)=f(x+1, y)+f(x-1, y)+f(x, y+1)+f(x, y-1)-4 f(x, y)
$$

\begin{tabular}{|c|c|}
\hline$\left(\begin{array}{ccc}1 & 1 & 1 \\
1 & -2 & 1 \\
-1 & -1 & -1\end{array}\right)$ & $\left(\begin{array}{ccc}1 & 1 & 1 \\
1 & -2 & -1 \\
1 & -1 & -1\end{array}\right)$ \\
\hline One-direction operator & Two-direction operator \\
\hline$\left(\begin{array}{ccc}1 & 1 & -1 \\
1 & -2 & -1 \\
1 & 1 & -1\end{array}\right)$ & $\left(\begin{array}{ccc}1 & -1 & -1 \\
1 & -2 & -1 \\
1 & 1 & 1\end{array}\right)$ \\
\hline Three-direction operator & Four-direction operator \\
\hline$\left(\begin{array}{lll}-1 & -1 & -1 \\
1 & -2 & 1 \\
1 & 1 & 1\end{array}\right)$ & $\left(\begin{array}{ccc}-1 & -1 & 1 \\
-1 & -2 & 1 \\
1 & 1 & 1\end{array}\right)$ \\
\hline Five-direction operator & Six-direction operator \\
\hline$\left(\begin{array}{lll}-1 & 1 & 1 \\
-1 & -2 & 1 \\
-1 & 1 & 1\end{array}\right)$ & $\left(\begin{array}{lll}-1 & 1 & 1 \\
-1 & -2 & 1 \\
-1 & -1 & 1\end{array}\right)$ \\
\hline Seven-direction operator & Eight-direction operator \\
\hline
\end{tabular}

Fig. 9 Eight-direction operator templates

In the digital images, Laplace values of calculation function also may be realized with the help of all kinds of templates. The basic requirements of templates are that the coefficient corresponding to the center pixels should be positive, while the coefficient corresponding to the pixels close to the center pixels should be negative; and their sum should be zero. The commonly used template is shown as the Figure 10.

This expression can be achieved by calculating the $\mathrm{F}$ and the convolution Template of Laplacian algorithm. The convolution template of Laplacian algorithm is:

$$
\left[\begin{array}{ccc}
0 & -1 & 0 \\
-1 & 4 & -1 \\
0 & -1 & 0
\end{array}\right]
$$

(a)

$$
\left[\begin{array}{ccc}
-1 & -1 & -1 \\
-1 & 8 & -1 \\
-1 & -1 & -1
\end{array}\right]
$$

(b)

Fig. 10 Laplacian operator template

Implementation of this algorithm mainly involves the image gray scale solving procedure and the nesting of a large loop and a small loop. The small loop is sequenced from 0 to 9 (because it is equivalent to the sequencing of the nine numbers of the first area where the whole image is divided into multiple $3 \times 3$ small areas, which is defined as a one-dimensional array), Then according to the operator to calculate the gray value of the point after convolution. The loop continues in the order. When it reaches Column 254, it will return to the next row to continue. It forms the outer large loop whose rows and columns start looping from 1 to 254 because the first row, column and last row and column are not considered ${ }^{[9]}$. As shown in Figure 11 is the image processed by Laplacian edge detection. 


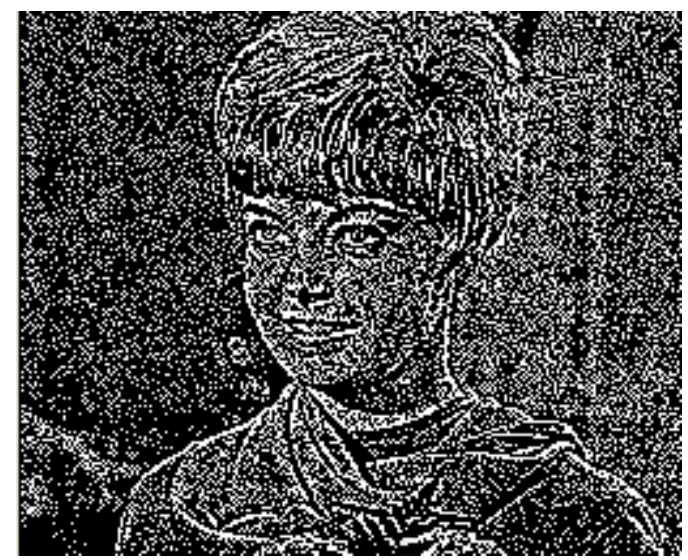

Fig. 11 the image processed by Laplacian edge detection

\section{Conclusion}

The edge of images is of great significance for human vision. The edge is both the end of one area and the start of another area. We can use this feature to segment images. In this Paper, the basic theory of image segmentation and image segmentation based on the edge introduced firstly ${ }^{[10]}$, A brief introduction of common edge detection methods and operators is completed secondly, Finally, The result of image segmentation with different operators on the same image are given. The effect of image segmentation is better by means of edge detection algorithm, which will lay a solid foundation for further research.

\section{Acknowledgment}

The work was financially supported by the surface project on science and technology research of the Education Department of Heilongjiang province(12521546) and the surface project on science and technology research of Jiamusi University(L2012-084).

\section{References}

[1] Jiang Xian-gang. Engineering Software Design of Digital Image Processing Based on Delphi [M]. Beijing: China Water Power Press, 2006.

[2] Zhang Yu-jin. A Review of Recent Evaluation Methods for Image Segmentation[C]. Kuala Lumpur,Malaysia:International Symposium on Signal Processing and Its Application,2001:13—16.

[3] Luo Lin.The Research of image segmentation method[D],WUHAN UNIVERSITY OF SICENCE TECHNOLOGY,2007.6

[4] Liu Yue,Ge Mao-song.The Research and implement of image segmentation method[J], JOURNAL OF QIQIHAER UNIVERSITY,2010.9

[5] Ma Yu-fei.Research on Image Edge Detection Based on Gradient Operator[D],XIDIAN UNIVERSITY,2012.3

[6] Didier Demigny. An Optima Linear Filtering for Edge Detection [J]. Image Processing,IEEE Transactions,2002,11(7):728-737.

[7] Pan Yuelong.Reseach and improvement on the algorithms of images edge detection based on gradient operators.Manufacturing Automation.2014.9

[8] Ma Lin, Yu Ning. Analysis of Digital Image Enhancement Algorithms [J], Technology \& Economy in Areas of Communications, 2011(1).

[9] Han Weifeng. Delphi 6.0 Program Design Course[M], Tsinghua University press,2002.

[10] Dong Ming. The Application And Research of Image Segmentation[D],Changchun University of Science and Technology,2009.6 